\title{
La comunicación de las enfermedades profesionales en la Comunidad Valenciana
}

\author{
Valentín Esteban Buedo ${ }^{a}$ Encarna Santolaria Bartoloméa
}

doi: 10.12961/aprl.2015.18.4.01

La Comunidad Valenciana dispone de un sistema de comunicación de sospechas de enfermedades profesionales (EEPP) desarrollado por la Dirección General de Salud Pública de la Conselleria de Sanidad Universal y Salud Pública, a partir del artículo 5 del Real Decreto 1299/2006, de 10 de noviembre, por el que se aprobó el actual cuadro de enfermedades profesionales en el sistema de la Seguridad Social ${ }^{1}$.

Forma parte del Sistema de Información Sanitaria y Vigilancia Epidemiológica Laboral (SISVEL), está regulado por la Orden 6/2012, de 19 de abril, del conseller de Sani$\operatorname{dad}^{2}$ y facilita a los facultativos de la Conselleria y a los de los servicios de prevención de riesgos laborales (SPRL) el cumplimiento de la obligación legal de comunicar a las Mutuas Colaboradoras de la Seguridad Social (Mutuas), empresas colaboradoras o al Instituto Nacional de la Seguridad Social (INSS), aquellas enfermedades que podrían ser calificadas como profesionales.

La comunicación se realiza vía electrónica, los facultativos de los SPRL, a través de un portal web específico, y los de la Conselleria por una integración del SISVEL con los sistemas de información asistenciales, especialmente el Sistema de Información Ambulatoria (SIA), que contiene la historia clínica personal. La Dirección General de Salud Pública actúa como organismo intermedio.

La Comunidad Valenciana tenía desde 1991 un sistema de vigilancia epidemiológica laboral basado en la metodología de sucesos centinela, con vigilancia de al menos cuatro problemas de salud y diferentes coberturas ${ }^{3}$. Durante el periodo 1997-2007, se investigaron 789 casos, procedentes mayoritariamente de Atención Primaria.

La implantación del nuevo sistema ha sido progresiva, comenzó en 2010 como experiencia piloto, conectando la aplicación informática en 20 centros de Atención Primaria, al menos uno por Departamento de Salud. En 2011 se extendió a los 770 Centros de Salud de Atención Primaria y se abrió el portal de los SPRL, con una lista reducida de 35 enfermedades. Actualmente, además de con SIA, hay integración con los principales sistemas hospitalarios, cubriendo al $100 \%$ de la población y todas las EEPP.

Para reforzar el uso del SISVEL se realizaron actividades informativas dirigidas a los profesionales sanitarios. En 2010 y 2011 se incluyeron objetivos de productividad relacionados con la participación en este sistema para los médicos de atención primaria y se publicó y distribuyó una guía para la comunicación de sospechas de enfermedades profesionales ${ }^{4}$. Estas acciones continúan, ahora con mayor énfasis para los profesionales sanitarios de atención especializada.

La comunicación de los posibles casos por los facultativos de la Conselleria de Sanidad se realiza mediante un sistema de alerta en la historia clínica informatizada, que los médicos deben responder. Se ha establecido un listado de 75 categorías diagnósticas. Ante cada uno de estos diagnósticos en mayores de 16 años, aparece una pantalla en la que se recuerda al facultativo la posibilidad del origen profesional de la enfermedad, permitiéndole su comunicación como sospecha de enfermedad profesional (EP), cumpliendo su deber legal establecido en el artículo 5 del citado RD 1299/2006. Si el médico no dispone de información suficiente para sustentar la sospecha del origen laboral de la enfermedad, el sistema le permite solicitar la investigación a la Unidad de Salud Laboral (USLA) del Centro de Salud Pública correspondiente. Para el resto de enfermedades profesionales existe un sistema alternativo de comunicación. a Dirección General de Salud Pública. Conselleria de Sanitat Universal i Salut Pública, Generalitat Valenciana.
Correspondencia:

Valentín Esteban

Servicio de Promoción de la Salud y Prevención en el Entorno Laboral Dirección General de Salud Pública

Conselleria de Sanitat Universal i Salut Pública

Avda. Cataluña 21; 46020-Valencia

esteban_val@gva.es 
Además, la aplicación recuerda al médico los criterios clínicos y de exposición mínimos que debe cumplir cada caso y según el problema de salud, para ser comunicado como sospecha de EP.

Esas comunicaciones se envían al SISVEL y éste remite telemáticamente los casos que el médico considera EP directamente a la Mutua correspondiente. Los casos en los que el médico no dispone de información suficiente, son estudiados, sobre todo en cuanto a los antecedentes de exposición laboral, por la USLA con la ayuda del trabajador y si es necesario del SPRL, comunicando los resultados a la Mutua, al trabajador y al médico notificador, que puede ir viendo desde SIA la información sobre las etapas y resultados del proceso, para informar al trabajador, cambiar el motivo de la baja, etc. Los médicos de la inspección médica y del INSS, para las determinaciones de contingencia, también tienen acceso al sistema online mediante perfiles de usuarios específicos.

Por tanto, el SISVEL, además de sistema de comunicación de sospechas de EEPP, actúa como sistema de apoyo para los médicos y establece una vía de comunicación entre el sistema sanitario público, el sistema de prevención de riesgos laborales y el sistema de atención y reconocimiento del daño laboral.

En cuanto a los resultados ${ }^{5}$, destacar que durante el año 2014 se recibieron 3480 sospechas de enfermedades profesionales, lo que supone una media de 290 comunicaciones al mes y un aumento del $15 \%$ respecto al año anterior. La mayoría de comunicaciones procedían de los facultativos de la propia Conselleria, con una tasa global de comunicación de 81.15 casos por cada 100.000 habitantes, cuando en 2011 esa tasa era de 22,63.

Por problemas de salud, más del $90 \%$ de los casos comunicados en 2014 eran del Grupo 2. Enfermedades provocadas por agentes físicos (anexo 1 del RD 1299/2006). Síndrome del túnel carpiano, epicondilitis y síndrome de la vaina de los rotadores del brazo agrupan el $78 \%$ de las enfermedades comunicadas. Las enfermedades respiratorias suponen un $4 \%$ de las sospechas recibidas.

En 2014 se comunicaron 2198 casos a las Mutuas, un 63\% de los recibidos en el SISVEL. Los principales motivos de no derivación a las Mutuas fueron no cumplir los criterios de exposición, trabajadores autónomos sin cobertura de contingencias profesionales y falta de respuesta del trabajador. Ese mismo año, los casos aceptados como contingencia profesional por las Mutuas fueron 702, un 34,4\% de los enviados, porcentaje similar al de años anteriores. El principal motivo para la no aceptación del caso como EP por parte de las Mutuas, que supone el 50\% del total de casos no considerados EP por las Mutuas, fue por incumplimiento de los criterios de exposición.

SISVEL se consolida y es reconocido como sistema eficaz para la detección de $\mathrm{EEPP}^{6}$ y está teniendo un impacto considerable en las estadísticas oficiales. Según los datos del Observatorio de Enfermedades Profesionales de la Seguridad Social, en 2013 se produjo un incremento del 64\% en las EEPP declaradas a CEPROSS desde la Comunidad Valenciana y en 2014 el aumento fue del 42\%. En esos dos últimos años el número de EEPP reconocidas en la Comunidad Valenciana se ha multiplicado por 2,5, aunque las cifras están aún muy por debajo de las estimaciones publicadas? $^{7}$.

SISVEL es útil para la detección precoz de algunos de estos daños a la salud de las personas que trabajan, permitiendo adelantar la adecuada atención y prevención. SISVEL es también de ayuda para las compensaciones económicas de los trabajadores afectados y del sistema sanitario público. Resulta muy útil para deslindar adecuadamente la responsabilidad financiera del tratamiento de estas enfermedades entre el Sistema de Seguridad Social (Mutuas o INSS) y la Conselleria de Sanidad Universal y de Salud Pública. Contribuye a mejorar el conocimiento de los problemas de salud y de exposición a riesgos de la población trabajadora. Permite realizar análisis epidemiológicos dirigidos a identificar los cambios en las tendencias de los problemas de salud y de riesgo de las personas que trabajan, así como otras investigaciones epidemiológicas, aportando información operativa para la planificación sanitaria eficaz y eficiente en materia de Salud Laboral y de Salud Pública en general.

En 2015 se trabaja para aumentar la sensibilidad del sistema para la detección de los problemas de salud con menor nivel de comunicación, para aumentar el número de comunicaciones desde los SPRL, y para mejorar la consistencia de las comunicaciones derivadas a Mutuas y aumentar su aceptación.

Entre las actuaciones previstas en este momento destaca la aplicación y ampliación de los "Criterios para la comunicación de sospechas y determinación de enfermedades profesionales", el refuerzo de la formación e información de los facultativos, la revisión y ampliación de los protocolos de comunicación y coordinación con el INSS y con las Mutuas y la mejora de la difusión de resultados.

En todo el proceso de desarrollo del SISVEL cabe resaltar la participación. El diseño, puesta en marcha y seguimiento se hace con la colaboración de representantes de la Administración Laboral, las asociaciones profesionales de Medicina y Enfermería del Trabajo, Medicina Familiar y Comunitaria, los agentes socio-laborales (organizaciones empresariales y sindicales), las Mutuas y los SPRL. Queremos destacar especialmente el buen nivel de colaboración de las 10 Mutuas con actividad en la Comunidad Valenciana, que han tenido que adaptar sus sistemas informáticos para recibir y responder al SISVEL. 


\section{BIBLIOGRAFÍA}

1. Real Decreto $1299 / 2006$ de 10 de noviembre, por el que se aprueba el cuadro de enfermedades profesionales en el sistema de Seguridad Social y se establecen los criterios para su notificación y registro. BOE 19 de diciembre de 2006.

2. Orden 6/2012, de 19 de abril, de la Conselleria de Sanidad, por la que se establece el procedimiento de comunicación de sospechas de enfermedades profesionales en la Comunitat Valenciana, a través del Sistema de Información Sanitaria y Vigilancia Epidemiológica Laboral. DOCV núm.6766/03-05-2012.

3. Servei de Salut Laboral. Informes de Salud, no 42. Programa Sistema de Información en Salud Laboral. Valencia: Dirección General de Salut Pública; 1998. Disponible en: http://publicaciones.san.gva.es/publicaciones/documentos/V.3877-1998.PDF

4. Santolaria E, Esteban V, Casanova, S. Guía para la comunicación de sospechas de enfermedades profesionales. Valencia: Generalitat. Conselleria de Sanitat; 2010 Disponible en: http://www.sp.san.gva.es/DgspPortal/docs/guia_com_enf_sos.pdf.
5. Esteban V, Santolaria E, Cadeddu P, Casanova S. Informe SISVEL. Principales resultados 2014 [Internet]. Conselleria de Sanitat; 2014 [citado 2015 Sep 22]; Disponible en: http://www.sp.san.gva.es/ DgspPortal/docs/INFORME_SISVEL_2014_PRINCIPALES_ RESULTADOS.pdf

6. Kieffer C. Nouveau rapport sur la déclaration des maladies professionnelles: problématique et bonnes pratiques dans cinq pays européens. Eurogip [Internet]. Enero 2015 [citado 2015 Sep 21]; 102/F. Disponible en: http://www.eurogip.fr/images/publications/2015/Rapport DeclarationMP_EUROGIP_102F.pdf

7. García AM, Gadea R. Estimaciones de incidencia y prevalencia de enfermedades de origen laboral en España. Aten Primaria. 2008; 40:43945.

8. Esteban Buedo V, Santolaria Bartolomé E, Casanova Vivas S, Ulldemolins Salvador C, García Layunta MD, Flores Reos E, Cadeddu Martín P. Criterios para la comunicación de sospechas y determinación de posibles enfermedades profesionales. Generalitat. Conselleria de Sanitat; 2014. 